\title{
Reducing the pressure drag of a D-shaped bluff body using linear feedback control
}

Received: 9 February 2016 / Accepted: 3 January 2017 / Published online: 18 January 2017

(c) The Author(s) 2017. This article is published with open access at Springerlink.com

\begin{abstract}
The pressure drag of blunt bluff bodies is highly relevant in many practical applications, including to the aerodynamic drag of road vehicles. This paper presents theory revealing that a mean drag reduction can be achieved by manipulating wake flow fluctuations. A linear feedback control strategy then exploits this idea, targeting attenuation of the spatially integrated base (back face) pressure fluctuations. Large-eddy simulations of the flow over a D-shaped blunt bluff body are used as a test-bed for this control strategy. The flow response to synthetic jet actuation is characterised using system identification, and controller design is via shaping of the frequency response to achieve fluctuation attenuation. The designed controller successfully attenuates integrated base pressure fluctuations, increasing the time-averaged pressure on the body base by $38 \%$. The effect on the flow field is to push the roll-up of vortices further downstream and increase the extent of the recirculation bubble. This control approach uses only body-mounted sensing/actuation and input-output model identification, meaning that it could be applied experimentally.
\end{abstract}

Keywords Bluff body aerodynamics $\cdot$ Flow control $\cdot$ Linear feedback control $\cdot$ Drag reduction

\section{Introduction}

Fluid flows around blunt bluff body shapes occur in a wide range of engineering applications. Of particular relevance for global $\mathrm{CO}_{2}$ emissions are road vehicles, for which the contribution of aerodynamic drag to driving resistance increases with speed, becoming the main component at highway speeds [1-3]. The aerodynamic drag of blunt bluff bodies is predominantly pressure drag (also known as form drag) as opposed to friction drag $[1,2]$. Boundary layer separation from the sharp corner at the top of the blunt rear face leads to a wake behind the body, in which the pressure is much lower than at the front.

The focus of this paper is reducing the pressure drag of bluff bodies using feedback control. We restrict attention to blunt bluff bodies, meaning that the rear face (base) of the body begins sharply, fixing the location of boundary layer separation. Consequently, attempts to reduce the pressure drag must focus on the wake flow itself, rather than manipulation of the separation location. Similar restrictions apply to squareback road vehicles. This is known as direct wake control [4].

Communicated by Peter Duck.

L. Dalla Longa · A. S. Morgans ( $\varangle)$

Department of Aeronautics, Imperial College London, SW7 2AZ London, UK

E-mail: a.morgans@imperial.ac.uk

Tel: +442075945053

J. A. Dahan

CD-adapco, 200 Shepherds Bush Road, W6 7NL London, UK 
Feedback control involves modifying the flow using an actuator, in response to measurements from a sensor $[4,5]$. This is in contrast to open loop control, where the actuator does not depend on measurements from a sensor $[4,5]$. The advantages of feedback control are that it allows the dynamics of a system to be modified, even allowing for the stabilisation of unstable flows [6,7]. It offers a theoretical framework for dealing with the effects of uncertainty and improving the energy efficiency of the control system and also offers the potential for automatic re-tuning as flow conditions change.

The body of literature addressing feedback control of blunt bluff body flows is significantly smaller than that which addresses either feedback control of bluff bodies with moveable separation lines, such as cylinders (where feedback control using an unsteady single degree-of-freedom sensor is more established), or passive or open loop control of blunt bluff bodies [4]. The examples which exist can be categorised into those addressing the backward-facing step geometry, with only a single shear layer, and those addressing a blunt bluff body geometry (square cylinders or D-shaped bodies), with interacting shear layers.

For a backward-facing step (BFS) geometry, the flow downstream of separation consists of a growing shear layer exhibiting the Kelvin-Helmholtz instability, a recirculation bubble, a bottom surface reattachment region and a subsequent redeveloping boundary layer [8]. The wake dynamics depends strongly on whether the boundary layer at separation is laminar, transitional or turbulent and whether the flow disturbances are 2-D or 3-D. For a 2-D laminar separating boundary layer, the flow is said to be an "amplifier" flow and exhibits only convective instability. Numerical simulations of such flows have been used as a test-bed in several active control studies, in which simplification of the Navier-Stokes equations was either used as a basis for feedback controller design [9,10], or input-output models were used as a basis for feedforward controller design [11,12]. For a BFS whose separating boundary layer has 3-D flow fluctuations at sufficiently high Reynolds number, the wake flow exhibits global instability and is an "oscillator" flow; most previous studies agree on the existence of a shedding/step mode and a lower frequency flapping or bubble pumping mode [13-15]. Previous numerical $[15,16]$ and experimental [8,17-19] feedback control studies have been performed; with the exception of [15], the sensor signal has been either based on wake velocity measurements or a measure of the reattachment length.

For blunt bluff bodies, such as the D-shaped body or square cylinder, above a critical Reynolds number an absolute wake instability $[8,20]$ generates alternating shedding of vortices at characteristic frequencies, known as a von Kármán vortex street. Numerical simulations of such flows have been as a test-bed for feedback control strategies, for example using Galerkin projection of the Navier-Stokes equations onto a lowdimensional subspace [21] or using on adjoint-based methods [22]. For the D-shaped bluff body, Henning and King [23] experimentally applied a gradient-based feedback scheme to increase the time-averaged base pressure of the turbulent flow around a D-body. Pastoor et al. [24] used both slope-seeking control and a gain/phase-shift controller targeting synchronisation of the two shear layers, achieving a 15\% drag reduction of the same experimental body. Stalnov et al. $[25,26]$ experimentally targeted reduced wake unsteadiness due to vortex shedding, using a phase-locked loop and reducing unsteadiness in the wake, while Flinois and Morgans [27] achieved full wake stabilisation at low Reynolds number, using balanced models derived from the unstable impulse response.

In summary, feedback control has been successfully applied to blunt bluff body flows in a limited number of studies. Feedback control based on models deduced from the governing Navier-Stokes equations appears feasible at very low Reynolds numbers $[9,21,22]$, but not under more realistic conditions, when either blackbox or reduced order models are necessary. In numerical simulations, optimal control in which the adjoint equations are solved can be used [9,22], but these methods cannot be extended to experiments. Finally, sensor signals based on flow field, rather than body-mounted sensing, can provide extra insights into the flow physics [19], but again will not be applicable in real moving-body experiments outside of the wind/water tunnel.

In the present study, numerical simulations of the flow past a D-shaped blunt bluff body are used as a control test-bed. The aim is to use linear feedback control to achieve an increase in mean pressure on the back face or base of the geometry, so as to achieve a pressure drag reduction. This approach has previously been successful for BFS flows [15]; the present study reveals some theory underpinning the link between mean drag and fluctuations, and extends application to bluff bodies exhibiting interacting shear layers. A notable feature of the present control strategy is that it should be implementable in real experiments outside of the wind tunnel, even though we are using computational flow simulations as a test-bed. The use of "adjoint" computational solvers is thus avoided, and body-mounted sensing and actuation are used. With the emphasis being on strategies which can be applied in real turbulent experiments, we design our controllers based on characterisation of the flow response to actuation via system identification, rather than any attempt to model the equations governing the system at hand, in this case the infinite dimensional Navier-Stokes equations. 
In Sect. 2, the link between mean aerodynamic drag and unsteady wake fluctuations is presented, along with the present feedback control approach. In Sect. 3, the target flow around a D-shaped bluff body with body-mounted sensing and actuation is described, along with the numerical methods used for its simulation. Section 4 presents the system identification, via unforced flow simulations, that is used to yield the relevant "black-box" model of the flow. This is used as the basis for designing a linear feedback controller, which is implemented and whose effect on the aerodynamic drag is studied. Finally, conclusions and outlook are presented in Sect. 5.

\section{Linear feedback control strategy}

\subsection{The relationship between mean drag and flow fluctuations}

The present drag reduction strategy is based on the premise that a reduction in the mean pressure drag can be achieved by manipulating temporal fluctuations of the flow. The link between wake fluctuations and mean aerodynamic drag has been observed in several experimental settings $[24,28]$ and was exploited using feedback control by Dahan et al. [15]. We now propose a theoretical underpinning for the link, using an unsteady control volume analysis.

A control volume containing incompressible flow around a bluff body is considered, as shown in Fig. 1. The flow is assumed longitudinal and undisturbed at the side, top and bottom boundaries, and the volume crosssectional area is constant, so that $\int_{S_{1}} \mathrm{~d} S=\int_{S_{2}} \mathrm{~d} S$. The flow entering is assumed steady, but is unsteady through the exit surface. The unsteady flow conservation equations, specifically the equations describing continuity of mass and $x$-momentum, for the control volume can be written,

$$
\begin{aligned}
\frac{\partial}{\partial t} \int_{V} \rho u(t) \mathrm{d} V & =-D(t)+p_{1} S_{1}-\int_{S_{2}} p_{2}(t) \mathrm{d} S+\rho U^{2} S_{1}-\int_{S_{2}} \rho u(t)^{2} \mathrm{~d} S+\int_{S_{2}} \tau_{x x} \mathrm{~d} S \\
U S_{1} & =\int_{S_{2}} u(t) \mathrm{d} S
\end{aligned}
$$

where $D(t)$ is the drag of the bluff body. The last term of Eq. (1) represents the viscous stress term acting in the $x$-direction on the exit surface. Although often neglected, it can be significant for near-wake flows [29].

The equations defining stagnation pressure are $p_{01}=p_{1}+\frac{1}{2} \rho U^{2}$ and $p_{02}(t)=p_{2}(t)+\frac{1}{2} \rho\left(u(t)^{2}+v(t)^{2}+\right.$ $\left.w(t)^{2}\right)$ [30]. For high Reynolds numbers flows, the viscous stress term can be assumed to be dominated by the Reynolds stress component, such that $\tau_{x x}=-\rho \overline{u^{\prime}(t)^{2}}$ [29]. It is then possible to express $D(t)$ as

$$
D(t)=\int_{\text {wake }} \Delta p_{0}(t) \mathrm{d} S+\frac{1}{2} \rho \int_{S_{2}}\left(U^{2}-u(t)^{2}+v(t)^{2}+w(t)^{2}-2 \overline{u^{\prime}(t)^{2}}\right) \mathrm{d} S+\rho \frac{\partial}{\partial t} \int_{V} u(t) \mathrm{d} V
$$

The term on the left-hand side represents overall aerodynamic drag. The first right-hand side term represents stagnation pressure loss in the wake; the exit and upstream stagnation pressures differ only over the wake component of the exit plane. The second term includes a longitudinal velocity deficit contribution, a vortexinduced drag contribution and a Reynolds stress contribution. The last term represents the rate of change

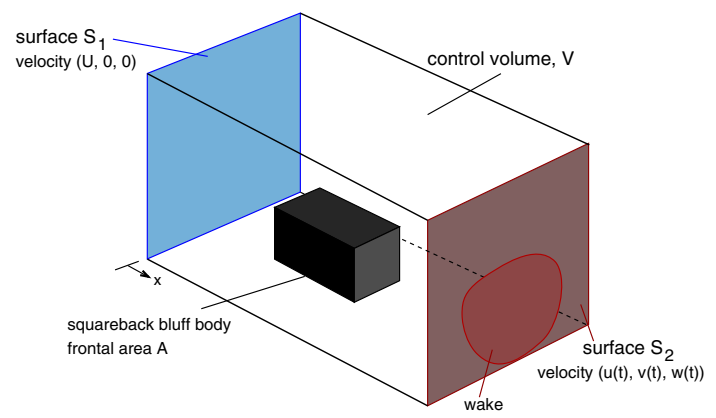

Fig. 1 Control volume analysis of the flow around a bluff body. The side, top and bottom walls are assumed impermeable 
of $x$-momentum in the control volume. The steady version of this equation is used widely in road vehicle aerodynamics as a tool for understanding drag [1,29,31].

Insights are drawn from the new, unsteady form of Eq. (3), when all variables are represented as the sum of a time-averaged ( ) and fluctuating ( ()' component. Because $D(t)$ depends linearly on stagnation pressure loss and $x$-momentum control volume unsteadiness, fluctuations in these will not alter the time-averaged $\bar{D}$. However, the term $\left(v^{\prime}(t)^{2}+w^{\prime}(t)^{2}-u^{\prime}(t)^{2}-2 \overline{u^{\prime}(t)^{2}}\right)$ comprises only velocity fluctuations, but due to the quadratic nature of its components has both a mean and fluctuating part. Reducing the size of this term reduces $\bar{D}$. That is, reducing velocity fluctuations normal to the streamwise direction, or increasing velocity fluctuations in the streamwise direction, results in a time-averaged drag reduction. This link seemingly provides an explanation for the impressive drag reductions achieved in high Reynolds number experiments using unsteady longitudinal slot jet actuation [32]. Of course, the link is a first-step approximation: altering the mean drag may in turn affect the mean and fluctuating values of other terms, complicating the final relation. It is our opinion that it is best used as an insight or guidance tool. The full unsteady version of this equation has not, to our knowledge, been published previously.

\subsection{Linear feedback control for fluctuation attenuation}

Our feedback control strategy exploits the link between mean drag and flow fluctuations. However, as the sensing of plane-integrated wake velocity fluctuations would require measurement at multiple locations in the flow field, we instead seek a body-mounted sensor-in our case the time varying value of some spatially integrated base pressure.

The issue of how to best capture the $\left(v^{\prime}(t)^{2}+w^{\prime}(t)^{2}-u^{\prime}(t)^{2}-2 \overline{u^{\prime}(t)^{2}}\right)$ term using body-mounted pressure sensing is an open question. Reducing the above bracketed term reduces the mean drag, $\bar{D}$, and also tends to reduce drag fluctuations, $D^{\prime}(t)$. Sensing of $D^{\prime}(t)$ (equivalent to temporal fluctuations in the base pressure force, assuming the skin friction drag is negligible and the pressure over the front of the body is steady) was the method used for BFS flows in Dahan et al. [15]. For flows exhibiting strong wake asymmetry, this dynamic term is likely to be better captured by the asymmetric component of the base pressure distribution; more formal correlation of base pressure modes to the above unsteady term will be the subject of future work.

Denoting the chosen sensor signal as $F_{\mathrm{p}}^{\prime}(t)$, the frequency domain models shown in Fig. 2 can be used. When actuating the flow with a varying slot jet velocity, $U_{j}^{\prime}(t), F_{\mathrm{p}}^{\prime}(t)$ can be assumed to vary both due to the actuation, $U_{j}^{\prime}(t)$, and due to other disturbances in the flow (for example boundary layer and shear layer disturbances), lumped together as "noise", $n(t)$. This captures the fact that even in the absence of actuation, the flow is fundamentally unsteady. Note that $s=i 2 \pi S t_{h}$ is the Laplace transform variable, where $S t_{h}$ represents non-dimensional frequency.

In the absence of feedback control, shown on the left of Fig. 2, the response of the sensor signal, $F_{\mathrm{p}}(s)$ is

$$
F_{\mathrm{p}}(s)_{\text {without control }}=H(s) n(s)+G(s) U_{j}(s)
$$

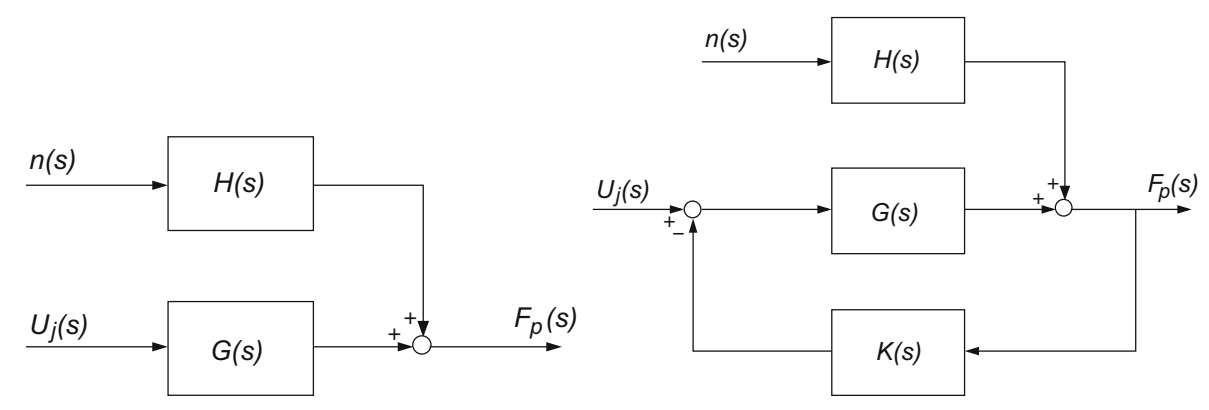

Fig. 2 Frequency domain models for the open loop system (left) and the feedback control system (right). $s=i 2 \pi S t_{h}$ is the Laplace transform variable, where $S t_{h}$ represents non-dimensional frequency 
By adding a feedback loop, in which the actuator responds to sensing of the base pressure force, $F_{\mathrm{p}}^{\prime}(t)$, via a control law, $K(s)$, shown on the right of Fig. 2, the response changes to

$$
F_{\mathrm{p}}(s)_{\text {with control }}=\frac{H(s) n(s)+G(s) U_{j}(s)}{1+G(s) K(s)}
$$

It can be seen that the ratio of sensor fluctuations with and without control is given by the magnitude of the sensitivity transfer function, $S(s)=1 /(1+G(s) K(s))$ [33].

$$
\frac{\left|F_{\mathrm{p}}(s)\right|_{\text {with control }}}{\left|F_{\mathrm{p}}(s)\right|_{\text {without control }}}=\frac{1}{|1+G(s) K(s)|}=|S(s)|
$$

Thus the aim of feedback control is to return a sensitivity magnitude smaller than unity, so that fluctuations in the chosen sensor signal are attenuated. It is known from Bode's integral law [34] that the sensitivity cannot be small over the entire frequency range, and so the dominant frequency range of the sensor fluctuations in the unforced flow is prioritised.

The control approach is then to identify $G(s)$, the transfer function describing the response of the sensor signal to actuation. Based on this, the feedback controller, $K(s)$, is designed to yield a sensitivity magnitude of less than unity, over the most dynamically relevant frequencies to the unforced flow. Note that although $H(s)$, the transfer function describing the response of the sensor signal to lumped "noise" is present in the model, its identification is never needed in order to achieve fluctuation attenuation.

\section{The D-shaped blunt bluff body flow}

The present work considers the simplified flow past a blunt bluff body of infinite spanwise extent, sometimes termed a D-shaped body. The Reynolds number based on the body height is $\operatorname{Re}_{h}=10,000$. The boundary layer at separation is laminar with $R e_{\theta}=270$; transition to turbulence occurs downstream of separation. The sensor signal is the unsteady component of the spatially integrated base pressure, taking the antisymmetric component in the $y$-direction, as shown in Fig. 3. It consists of taking the integrated value of the pressure on the base and counting as negative the values on the lower half. This accounts for the antisymmetric nature of the vortex shedding. Actuation is via slot-jet velocity forcing with zero mean, located just ahead of separation at an angle of $45^{\circ}$. This is out of phase on the upper and lower edges and is constant in the span-wise direction. Our aim is to develop and demonstrate a feedback control strategy that increases the mean base pressure, via targeting attenuation of fluctuations in the sensor signal.

\subsection{Incompressible large-eddy simulations}

Flow simulations are performed using an in-house large-eddy simulation (LES) code, "Stream-LES", which solves the incompressible Navier-Stokes equations $[35,36]$. The code solves for the velocity field via a colocated finite-volume method on a cartesian grid, using a second-order accurate scheme for the fluxes [37]. The solution is time-marched via a third-order Gear-like fractional-step method. Pressure-correction is obtained by solving the Poisson equation using a multigrid successive over-relaxation technique. The code is fully
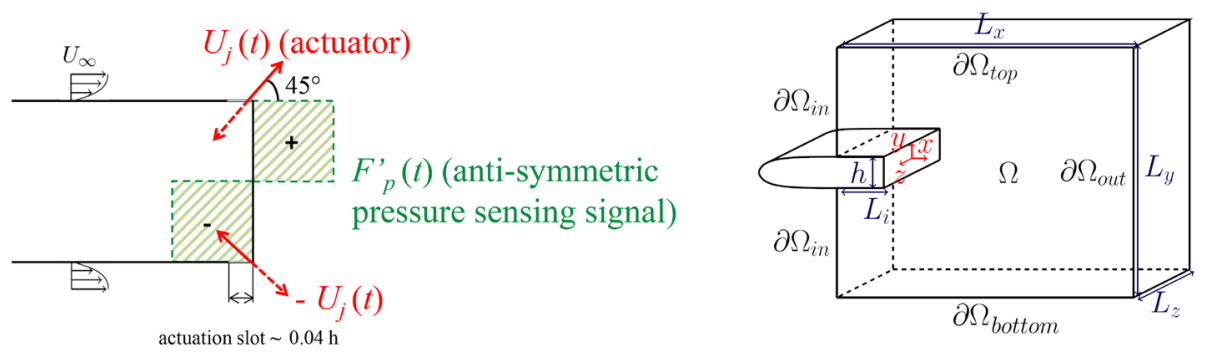

Fig. 3 Left side view of the setup showing actuation and sensing, flow left to right (the front part of the D-body is not shown). Right the computational domain 
parallelised using message passing interface. The subgrid-scale stresses are simulated by the WALE (walladapting local eddy-viscosity) model [38]. This reproduces the cubic wall-asymptotic behaviour of the eddy viscosity and returns lower values of this viscosity compared to the Smagorinsky model [36]. For all cases, actuation is performed with a simple "top hat" slot jet velocity profile [14] across the last five points of the grid upstream of separation, with slot width $s=0.04 h$, extending along the full span on both top and bottom.

The computational domain is shown diagrammatically in Fig. 3. It has dimensions $\left(L_{i}, L_{x}, L_{y}, L_{z}\right)=$ $(4 h, 24 h, 9 h, 4 h)$. The inflow boundary condition, on both the upper and lower surfaces of the body, is a laminar Blasius boundary layer profile of thickness $\delta=0.1 \mathrm{~h}$, superimposed with random perturbations to encourage transition to turbulence. The top and bottom boundaries have free-slip conditions, with a periodic condition imposed in the spanwise direction. Consequently, the flow is statistically homogeneous in the spanwise direction and the time averaging is coupled with a spanwise averaging to accelerate statistical convergence. Grid checks were performed by increasing the resolution in each direction until consistency was achieved. The final mesh comprises 9.4 million cells, with local refinement near walls and in the upper and lower actuation region. The spanwise spacing was $\Delta z^{+}=12.4$ and $\Delta y_{\max }^{+}<1$ along the body.

\section{Implementation and results}

\subsection{Unforced flow field and integrated base pressure spectrum}

The time-averaged streamlines and integrated base pressure spectra (both for the base pressure force and the antisymmetric component) for the unforced flow are shown in Fig. 4. The base pressure spectra exhibit a large narrow peak at $S t_{h} \approx 0.24$, corresponding to the vortex shedding mode. This frequency agrees well with the literature $[8,24]$, and the first harmonic is also observed. The antisymmetric component of the base pressure is seen to exhibit the vortex shedding mode more strongly, confirming that as a sensor signal, it is capturing the wake fluctuations efficiently. This spectrum identifies the frequency range over which feedback control needs to provide attenuation.

Figure 5 shows vorticity magnitude and instantaneous isosurfaces of pressure coefficient. The large-scale spanwise vortices, or rollers, that are shed downstream of the bluff body are evident, arranged in the well-known von Kármán vortex street pattern. These vortices are produced by the alternate roll-up of the upper and lower shear layers. Isosurfaces of negative pressure coefficient $C_{\mathrm{p}}=-0.1$, extracted from the same snapshot, are shown in Fig. 5. The large-scale vortical structures resulting from the roll-up are seen to have a low pressure core.

\subsection{System identification}

The aim of feedback control is to attenuate temporal fluctuations in the antisymmetrically integrated base pressure. The linear feedback controller, $K(s)$, will be designed so as to shape the gain of the sensitivity transfer function, $S(s)=1 /(1+G(s) K(s))$, ensuring its magnitude is less than unity over the dynamically
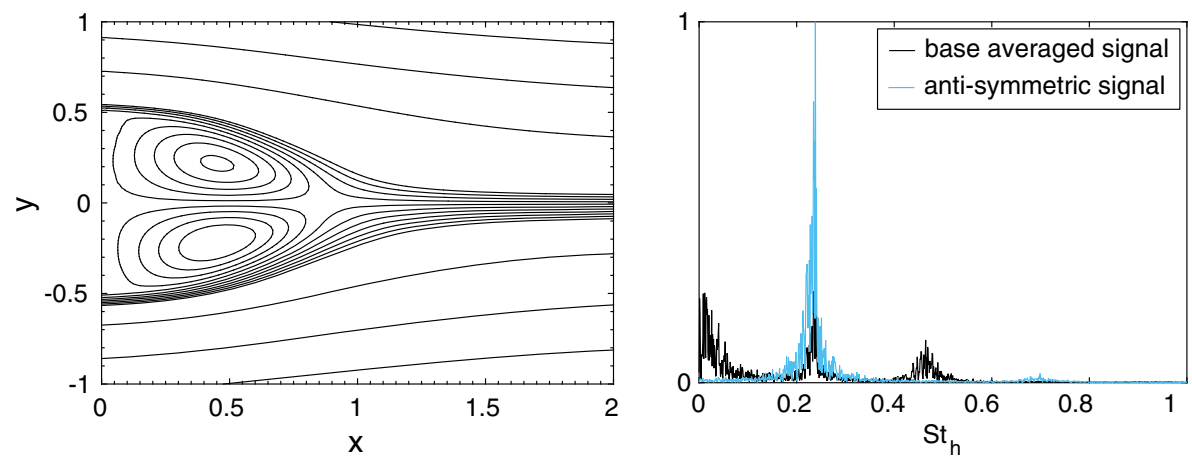

Fig. 4 Time-averaged streamlines (left) and spectra of the normalised spatially integrated base pressure (right) for the unforced flow 
relevant frequencies. To accomplish this, it is necessary to characterise $G(s)$, the open loop response of the system to actuation.

The flow is governed by non-linear fluid mechanics. However, we make (and later check) the assumption of dynamic linearity - that the response of the change in the flow due to actuation is approximately linear. When a purely harmonic actuation signal is applied, the sensor signal eventually settles into a response at the same frequency. The gain and phase shift of the sensor signal compared to the actuation are then used to deduce the gain and phase of $G\left(i S t_{h}\right)$ [39]. Because the forcing frequency is clearly identifiable in the very clean output sensing signal, the integration of the product of the output signal and the relevant harmonic over at least 15 periods was sufficient to extract the gain and phase shift. No further signal processing tools were required. This is repeated across different frequencies, spanning the range for which the unforced flow exhibits dynamics in Fig. 4. The assumption of dynamic linearity is checked by ensuring that (1) the main flow response is at the forcing frequency with no significant scattering into harmonics and (2) there is no significant dependence of the gain and phase shift on forcing amplitude.

The forcing frequency is found to dominate the response across the range of forcing frequencies applied. The amplitude and phase variations of $G\left(i S t_{h}\right)$ as a function of non-dimensional frequency, $S t_{h}$, are shown in Fig. 6, with the effect of forcing amplitude included. It can be seen that the dynamic response varies little with the forcing amplitude, $A_{j}$, for the forcing range considered. Hence as long as control actuation remains within this range, the assumption of dynamic linearity appears to be justified. The MATLAB command "fitfrd" is used to fit the frequency response data with a state-space model, using the "average" frequency response across the three forcing amplitudes in the fitting procedure; a fifth order fit is chosen, as shown in Fig. 6.

\subsection{Controller design}

Design of the linear feedback controller, $K(s)$, is performed manually in the frequency domain, with the objective of yielding a sensitivity magnitude less than unity over dominant frequency range of the spectrum in Fig. 4. Controller iteration to achieve a good compromise between the size and bandwidth of sensitivity, closed loop stability and robustness to model uncertainty at high frequencies was performed [40]. The unforced
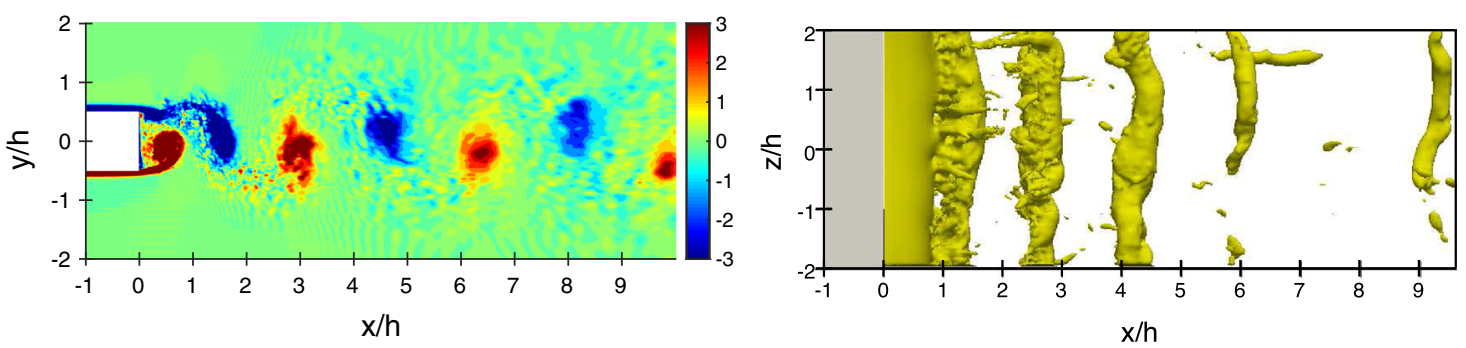

Fig. 5 Left vorticity, $\omega_{z}$, side view at $z / h=0$. Right instantaneous isosurfaces of pressure coefficient $C_{\mathrm{p}}=-0.1$, bottom view at $y / h=0$
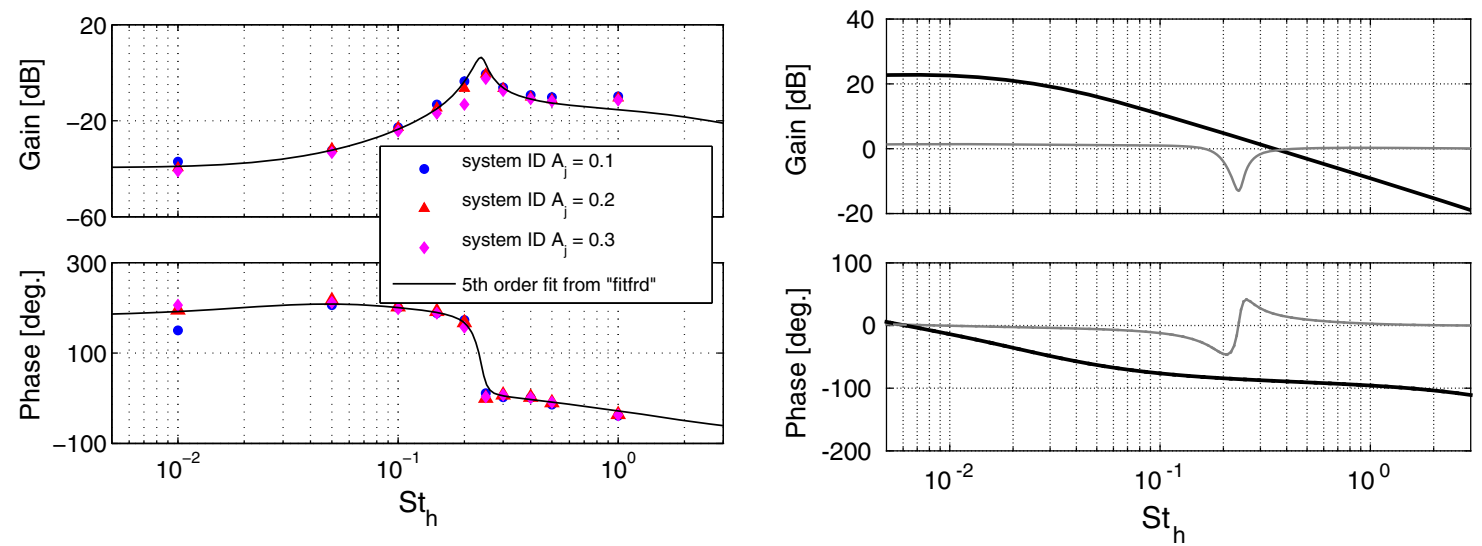

Fig. 6 Left system identification of $G\left(i S t_{h}\right)$. Right controller design: $K\left(i S t_{h}\right)\left(\right.$ black) and $S\left(i S t_{h}\right)$ (grey) 

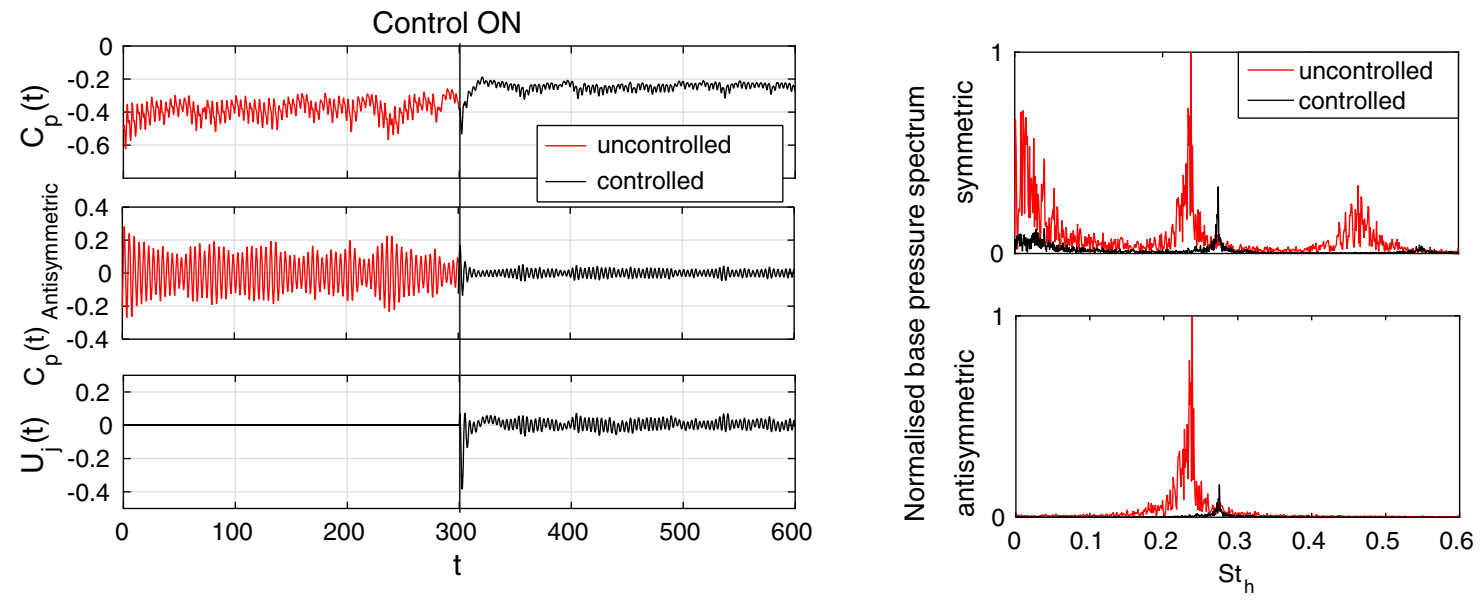

Fig. 7 Effect of control, showing both the antisymmetric and symmetric components of the spatially integrated base pressure. Time domain (left), and frequency domain (right). Control is switched on at $t=300$
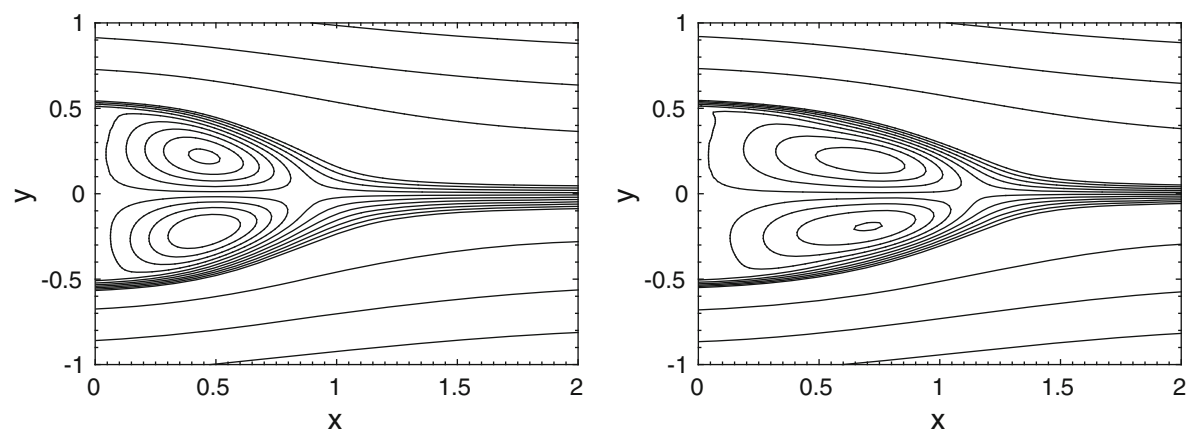

Fig. 8 Contours of time-averaged stream function (left) without control and (right) with control

sensor spectrum consists of a main peak, and so classical loop-shaping via a fourth order controller is used to shape the gain of the sensitivity function [40]. The resulting controller is $K(s)=2.2 \times 10^{4} s /\left(s^{4}+200 s^{3}+\right.$ $\left.1 \times 10^{4} s^{2}+1600 s+15\right)$. Its frequency response, along with the resulting sensitivity transfer function, are shown on the right of Fig. 6 . The sensitivity magnitude is less than unity (i.e. $0 \mathrm{~dB}$ ) for $0.15 \lesssim S t_{h} \lesssim 0.3$; hence, the dominant frequencies in Fig. 4 should be attenuated by the action of feedback control.

\subsection{Effect of feedback control on the base pressure}

The controller was implemented in discrete-time format in the flow simulations, with the discrete-time controller coefficients obtained via a bilinear transform. The results are shown in Fig. 7, where the spatially integrated base pressure has been non-dimensionalised as a pressure coefficient, $C_{\mathrm{p}}(t)$. The controller achieves its primary objective of reducing the antisymmetric component of the base pressure fluctuations over their most dynamic frequency range; the symmetric base pressure fluctuations are also attenuated. The ultimate and indirect aim of feedback control was to increase the base pressure. This was successful; the mean pressure force is increased by $38 \%$.

\subsection{Effect of feedback control on the flow field}

The changes to the flow field accompanying effective feedback control are shown in Figs. 8 and 9. The feedback control acts to delay the roll-up of the shear layer vortices. Shed vortices are pushed further downstream; consequently, the recirculation region is extended in the $x$-direction. This is a similar observation to the 2-D BFS in [15], where the effect of control was also to push the roll-up of the shed vortices further downstream. 

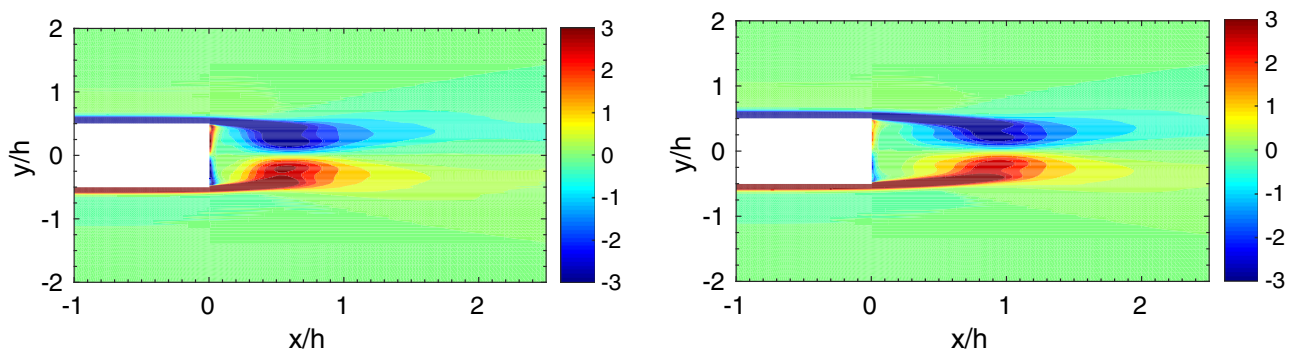

Fig. 9 Contours of time-averaged vorticity $\omega_{z}$, (left) without control and (right) with control
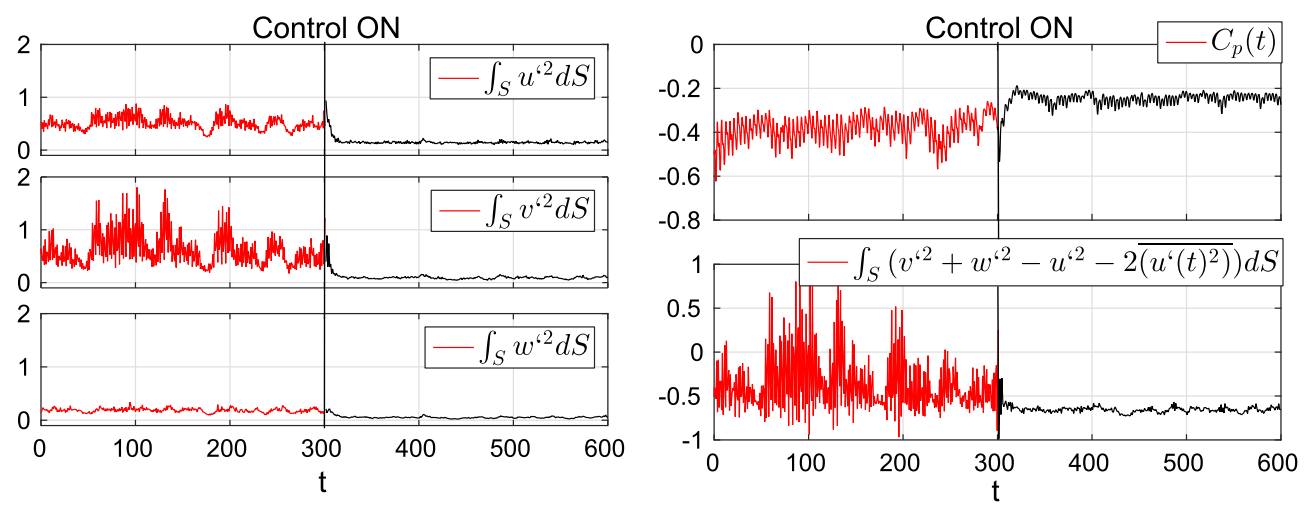

Fig. 10 Effect of control, integrated values of squared velocity fluctuation components over a plane $x=0.5 h$ downstream of the body (left). Comparison between the sum of the plane integrated velocity fluctuations and the base pressure signal (right). Control is switched on at $t=300$

To verify the unsteady wake analysis findings presented in Sect. 2 , the relevant squared velocity fluctuations integrated over a plane $x=0.5 \mathrm{~h}$ downstream of the body are shown in Fig. 10, both prior to and after feedback control is applied.

It is clear that although the control target is reducing spatially integrated base pressure fluctuations, it also indirectly reduces the wake flow velocity fluctuations term identified in Sect. 2.1. This is consistent with the reduction in the pressure drag observed.

Finally, as it is net energy gain that is important in drag reduction, the efficiency of the control method has been evaluated. The power injected into the jet fluid, $P_{\text {jet }}$, is compared to the power saving associated with the drag reduction, $P_{\text {saved. }}$. The relevant expressions are

$$
P_{\text {jet }}=\frac{1}{\eta_{\text {jet }}} \rho \frac{U_{\text {jet }}^{3} S_{\text {jet }}}{2} \quad P_{\text {saved }}=\Delta F_{x} U
$$

where $\eta_{\text {jet }}$ is actuator efficiency, $U_{\text {jet }}$ jet velocity, $U$ free stream velocity, $S_{\text {jet }}$ slot-jet area and $\Delta F_{x}$ the drag reduction. Initially, the actuator delivers a fairly large amount of momentum to obtain control of the flow, and the momentum coefficient peaks at $\max \left(C_{\mu}\right)=s \max \left(U_{\text {jet }}\right)^{2} /\left(h U^{2}\right)=1.18 \times 10^{-2}$ where $\mathrm{s}$ is the slot width and $\max \left(U_{\text {jet }}\right)$ the maximum jet velocity. However, once the controlled state has been achieved, much less actuation is required to maintain the low drag state and $C_{\mu}=s U_{\text {jet,rms }}^{2} /\left(h U^{2}\right)=2.60 \times 10^{-4}$, Thus if we consider an ideal synthetic jet (i.e. $\eta_{\text {jet }}=1$ ) we obtain $P_{\text {saved }} / P_{\text {jet }}=8947$. This then provides a lower bound on the required actuator efficiency for net energy gain as being $0.01 \%$, which is easily achievable by most of the synthetic jets devices available [41].

\section{Conclusions}

The present work has presented a concept for reducing the aerodynamic drag of bluff body flows, based on sensing and manipulating only fluctuating flow quantities. This is underpinned by theory and facilitates the use of linear feedback control strategies which target attenuation of temporal base pressure fluctuations. 
The flow over a simple blunt bluff body (a "D-shaped body"), with upper and lower edges exhibiting interacting shear layers, was considered. Actuation in the form of zero-mean slot jet forcing at $45^{\circ}$ just ahead of separation was used, and a relevant form of the spatially integrated base pressure was used for sensing. Large eddy simulations were used as a test-bed. The response to actuation was characterised via harmonic forcing system identification, and the controller was designed in the frequency domain, so as to give fluctuation attenuation over the frequency range in which the flow dynamics were concentrated.

Upon implementing the feedback controller, it achieved both its direct aim of attenuating base pressure fluctuations, and its indirect aim of increasing the mean base pressure, in this case by $38 \%$. The control strategy is based only on body-mounted sensing and actuation, does not require solving the adjoint equations, and is not based on approximations of the infinite-dimensional governing flow equations. Thus the strategy would apply to moving bluff body experiments. An important open question is the best way in which to target the fluctuating terms which are linked to mean drag, using only body-mounted sensing, and this will form the basis of further studies.

Acknowledgements This work used both the Imperial College High Performance Computing service and ARCHER (the UK High Performance Computing resource). Financial support from the Engineering and Physical Sciences Research Council, the Imperial College $\mathrm{PhD}$ Scholarships and Renault is gratefully acknowledged.

Open Access This article is distributed under the terms of the Creative Commons Attribution 4.0 International License (http:// creativecommons.org/licenses/by/4.0/), which permits unrestricted use, distribution, and reproduction in any medium, provided you give appropriate credit to the original author(s) and the source, provide a link to the Creative Commons license, and indicate if changes were made.

\section{References}

1. Hucho, W.H.: Aerodynamics of Road Vehicles. SAE International, Sydney (1998)

2. Choi, H., Lee, J., Park, H.: Aerodynamics of heavy vehicles. Ann. Rev. Fluid Mech. 46, 441 (2014)

3. Seifert, A., Stalnov, O., Sperber, D., Arwatz, G., Palei, V., David, S., Dayan, I., Fono, I.: Large trucks drag reduction using active flow control. In: Browand, F., McCallen, R., Ross J. (eds.) The Aerodynamics of Heavy Vehicles II: Trucks, Buses, and Trains. Lecture Notes in Applied and Computational Mechanics, vol. 41, pp. 115-133. Springer, Berlin (2009)

4. Choi, H., Jeon, W.P., Kim, J.: Control of flow over a bluff body. Ann. Rev. Fluid Mech. 40, 113 (2008)

5. Gad-el-Hak, M.: Flow Control-Passive, Active and Reactive Flow Management. Cambridge University Press, Cambridge (2000)

6. Cattafesta, L.N., Song, Q., Williams, D.R., Rowley, C.W., Alvi, F.S.: Active control of flow-induced cavity oscillations. Prog. Aerosp. Sci. 44, 479 (2008)

7. Dowling, A.P., Morgans, A.S.: Feedback control of combustion oscillations. Annu. Rev. Fluid Mech. 37, 151 (2005)

8. Henning, L., King, R.: Robust multivariable closed-loop control of a turbulent backward-facing step flow. J. Aircr. 44(1), 201 (2007)

9. Choi, H., Jeon, W.P., Kim, J.: Instantaneous control of backward facing step flows. Appl. Numer. Math. 31, 133 (1999)

10. Alam, M.R., Liu, W., Haller, G.: Closed-loop separation control: an analytic approach. Phys. Fluids 18(043601), 1 (2006)

11. Hervé, A., Sipp, D., Schmid, P.J., Samuelides, M.: A physics-based approach to flow control using system identification. J. Fluid Mech. 702, 26 (2012)

12. Juillet, F., Schmid, P.J., Huerre, P.: Control of amplifier flows using subspace identification methods. J. Fluid Mech. 725, 522 (2013)

13. Hasan, M.A.Z., Khan, A.S.: On the instability characteristics of a reattaching shear layer with nonlaminar separation. Int. J. Heat Fluid Flow 13(3), 224 (1992)

14. Leschziner, M.A., Lardeau, S.: Simulation of slot and round synthetic jets in the context of boundary layer separation. Phil. Trans. R. Soc. A 369, 1495 (2011)

15. Dahan, J.A., Morgans, A.S., Lardeau, S.: Feedback control for form-drag reduction on a bluff body with a blunt trailing edge. J. Fluid Mech. 704, 360 (2012)

16. Kang, S., Choi, H.: Suboptimal feedback control of turbulent flow over a backward facing step. J. Fluid Mech. 463, 201 (2002)

17. Becker, R., Garwon, M., Guknecht, C., Barwolff, G., King, R.: Robust control of separated shear flows in simulation and experiment. J. Process Control 15(6), 691 (2005)

18. Marrot, F., Gajan, P., Pauzin, S., Simon, F.: Experimental application of an active control loop on a backward-facing step. AIAA J. 43(6), 1176 (2005)

19. Gautier, N., Aider, J.L.: Control of the separated flow downstream of a backward-facing step using visual feedback. Proc. R. Soc. A 469(2160), 1 (2013)

20. Huerre, P., Monkewitz, P.A.: Local and global instabilities in spatially developing flows. Annu. Rev. Fluid Mech. 22, 473 (1990)

21. Weller, J., Camarri, S., Iollo, A.: Feedback control by low-order modelling of the laminar flow past a bluff body. J. Fluid Mech. 634, 405 (2009)

22. Camarri, S., Iollo, A.: Feedback control of the vortex shedding instability based on sensitivity analysis. Phys. Fluids 22(9), 1 (2010) 
23. Henning, L., King, R.: Drag reduction by closed-loop control of a separated flow over a bluff body with a blunt trailing edge. In: Proceedings of the 44th IEEE Conference on Decision and Control, pp. 494-499 (2005)

24. Pastoor, M., Henning, L., Noack, B.D., King, R., Tadmor, G.: Feedback shear layer control for bluff body drag reduction. J. Fluid Mech. 608, 161 (2008)

25. Stalnov, O., Palei, V., Fono, I., Cohen, K., Seifert, A.: Experimental estimation of a D-shaped cylinder wake using bodymounted sensors. Exp. Fluids 42, 531 (2007)

26. Stalnov, O., Fono, I., Seifert, A.: Closed-loop bluff-body wake stabilization via fluidic excitation. Theor. Comput. Fluid Dyn. 25, $209(2011)$

27. Flinois, T.L.B., Morgans, A.S.: Feedback control of unstable flows: a direct modelling approach using the eigensystem realisation algorithm. J. Fluid Mech. 793, 41 (2016)

28. Heenan, A.F., Morrison, J.F.: Passive control of pressure fluctuations generated by separated flow. AIAA J. 36(6), 1014 (1998)

29. van Dam, C.P.: Recent experience with different methods of drag prediction. Prog. Aerospa. Sci. 35(8), 751 (1999)

30. Greitzer, E.M., Tan, C.S., Graf, M.B.: Internal Flow: Concepts and Applications. Cambridge University Press, Cambridge (2004)

31. Fourrié, G., Keirsbulck, L., Labraga, L., Gilliéron, P.: Bluff-body drag reduction using a deflector. Exp. Fluids 50(2), 385 (2011)

32. Oxlade, A.R., Morrison, J.F., Qubain, A., Rigas, G.: High-frequency forcing of a turbulent axisymmetric wake. J. Fluid Mech. 770, 305 (2015)

33. Franklin, G.F., Powell, J.D., Emami-Naeini, A.: Feedback Control of Dynamic Systems, 4th edn. Prentice Hall, Upper Saddle River (2002)

34. Aström, K.J., Murray, R.M.: Feedback Systems: An Introduction for Scientists and Engineers. Princeton University Press, Princeton (2008)

35. Lardat, R., Leschziner, M.A.: A Navier-Stokes solver for LES on parallel computers. Technical report, Department of Mechanical Engineering, UMIST (1998)

36. Temmerman, L., Leschziner, M.A., Mellen, C.P., Fröhlich, J.: Investigation of subgrid-scale models and wall-functions approximations in large eddy simulation of separated flow in a streamwise periodic channel constriction. Int. J. Heat Fluid Flow 24, 157 (2003)

37. Fishpool, G.M., Leschziner, M.A.: Stability bounds for explicit fractional-step schemes for the Navier-Stokes equations at high Reynolds number. Comput. Fluids 38, 1289 (2009)

38. Nicoud, F., Ducros, F.: Subgrid-scale stress modelling based on the square of the velocity gradient tensor. Flow. Turbul. Combust. 62, 183,200 (1999)

39. Ljung, L.: System Identification: Theory for the User. Prentice-Hall, Upper Saddle River (1999)

40. Zhou, K., Doyle, J.C., Glover, K.: Robust and Optimal Control. Prentice Hall, Englewood Cliffs (1996)

41. Seifert, A.: Closed-loop active flow control systems: actuators. In: King, R. (ed.) Active Flow Control, pp. 85-102. Springer, Berlin (2007) 